cardiologist. The study period was 6 months (19th of September 2016 - 15th of February 2017) confidence interval $99 \%$ and confidence width, 01.

Results Among 2400 pupils 10 new cases of CHD were founded giving the prevalence of 4,2:1000. Male:Female ratio $1: 1.4$, with ASD being the commonest representing 5 cases $(, 21 \%)$ giving the prevalence of 2:1000, Dextrocardia 2 cases (,083\%) prevalence of, 8:1000, MVP 2 cases (,083\%) with prevalence of, 83:1000 and Dilated Aortic roots 1 case $(, 045 \%)$ giving the prevalence of, 45:1000. The innocent murmurs were common representing 18\%(431 children) of study group. And the statistical tests revealed associations between CHD and sex (p-value, 042) And also there is association between CHD and age ( $\mathrm{p}$-value, 003) and school absence ( $\mathrm{p}$ value, 035).

Conclusion CHD prevalence is almost as what mentioned in literature, innocent murmurs are not uncommon, as it is asymptomatic and not affecting pupils general health and no serious complications has been detected clinically or by ECHO, So early screening and involving of health system and governmental recruitment for this sake is doubtful and may not be cost effective in such a depleted 3rd world country like Sudan but clinical examination and oximetry screening may be of benefit as preschool assessment tool.

\section{G27(P) 22Q11 DELETION SYNDROME - IMPROVING INITIAL INVESTIGATIONS AT A TERTIARY CARDIAC CENTRE}

I Sohal, AJ Pearce, M Harris. Paediatric Cardiology, Birmingham Children's Hospital, Birmingham, UK

\subsection{6/archdischild-2020-rcpch. 16}

Aims 22q11 Deletion Syndrome (22q11DS) or DiGeorge syndrome is commonly first considered and diagnosed at cardiac centres due to its association with several forms of congenital heart disease. Due its multi-system involvement, it is important to screen children with 22q11DS for common problems early and refer promptly. The aims of this project were to: 1. Audit our practice against any national standards of initial investigations. 2. Create a local pathway and checklist for initial management of suspected or confirmed DiGeorge syndrome.

Methods We used the MaxAppeal (www.maxappeal.org.uk) consensus document on 22q11DS to formulate a list of standards to retrospectively audit against. We then used our local electronic patient record system to search for all children who had been admitted under cardiology with a diagnosis of 22q11DS syndrome between 2014-2018. We searched our laboratory, radiology and other electronic patient record system to record if and when they had certain investigations. We excluded patients whose care was primarily under another hospital.

Results We found 46 patients diagnosed between 20142018 but 21 of these had their care under another hospital. 23 out of the remaining 25 had a period of inpatient care and 17 of the 25 were diagnosed with 22q11DS during their first admission to hospital. 4/25 were diagnosed antenatally. We performed well on ensuring all our children had a baseline full blood count with white cell differentials and plasma calcium. However, only $7 / 25$ had a thyroid function test. Unsurprisingly all 25 patients had a full cardiac examination and ECHO. Renal ultrasound was performed in $17 / 25$ of our patients. The largest room for improvement was in baseline immunology investigations; only $7 / 25$ had lymphocyte phenotyping and $6 / 25$ had immunoglobulins tested.

Conclusion The data shows that 22q11DS is a not uncommon clinical diagnosis in the tertiary cardiac centre. There are multiple considerations in management and investigations and our retrospective audit shows that there is room for improvement, particularly in immunological investigation. We are developing a proforma for suspected and confirmed 22q11DS and will be presenting this locally for approval and dissemination.

\section{G28(P) ABSTRACT WITHDRAWN}

\section{G29(P) IDIOPATHIC COMPLETE HEART BLOCK OF CHILDHOOD: CASE REPORT}

${ }^{1} \mathrm{O}$ Nwanko, ${ }^{2} \mathrm{~L}$ Starling, ${ }^{1} \mathrm{P}$ Nair. ${ }^{1}$ Paediatric Department, Bedford Hospital NHS Trust, Bedford, UK; ${ }^{2}$ Great Ormond Street Hospital, London, UK

\subsection{6/archdischild-2020-rcpch.17}

Introduction Paediatric complete heart blocks are predominantly congenital with cases often diagnosed in utero, at birth or usually within the first 1 month of life. This is secondary to immune mediated injury of the conduction system due to transplacental passage of maternal anti-SSA/Ro-SSB/La antibodies. Childhood AV (atrioventricular) block is one which is diagnosed between 1 month and 18 years of age, which could be due to maternal antibodies, congenital heart defect, iatrogenic or due to underlying genetic causes. Idiopathic complete heart block of childhood is a rare entity with scarce case reports.

Case Report We report a case of 13 month old previously fit boy diagnosed with idiopathic complete AV block. $\mathrm{He}$ was admitted at 13 months of age with an episode of febrile convulsion secondary to throat infection. He was noted to have a low heart rate of around 50 beats per minute. There was no family history of cardiac problems or need for pacemakers. An ECG (electrocardiogram) done showed evidence of complete heart block with QTc of 500 ms. He was otherwise hemodynamically stable with normal blood parameters. His echocardiography showed mild dilatation of both ventricles with overall good function \& structurally normal arrangements. His blood tests showed normal thyroid function, kidney function, iron levels, vitamin D levels, creatine kinase levels and auto-antibody screen. The anti Ro-La antibodies were negative in both the child and his mother. The genetic screening for inherited causes of complete heart block was also negative. He remains stable with close monitoring by the cardiac team with no evidence of arrhythmia or current need for pacemaker at 2 years of age. He has a sibling who was also screened and investigations were normal.

Discussion This case currently falls in the idiopathic AV block of childhood of which there are only scarce case reports worldwide. We currently do not know about the etiology or clinical course of these cases. Future genetic studies might reveal an underlying genetic cause in these cases. Further 